\title{
Opciones terapéuticas para el defecto de cicatriz uterina
}

\author{
Angie Carolina Carreño-Martínez* \\ Janer Sepúlveda-Agudelo**
}

\begin{abstract}
*Estudiante de XII semestre. Escuela de Medicina. Facultad de Salud. Universidad Industrial de Santander. Bucaramanga. Santander. Colombia. **Médico Gineco-obstetra. Especialista en Cirugía Endoscópica Ginecológica. Profesor Asociado. Departamento de Gineco-obstetricia. Universidad Industrial de Santander. Grupo de Investigación GINO. Bucaramanga. Santander. Colombia.

Correspondencia: Srta. Angie Carolina Carreño Martínez. Dirección: Cra 12\#200-105 Floridablanca. Santander. Colombia. Correo electrónico: angieccm@outlook.com
\end{abstract}

Resumen

La cicatriz uterina es un defecto en la pared anterior del miometrio secundario a la cesárea. Su prevalencia es muy variable, Ilegando hasta el $84 \%$ en algunos estudios, y aumenta según el número de cesáreas previas. La mayoría de las pacientes pueden ser asintomáticas, pero cuando se manifiesta es principalmente con hemorragia uterina anormal. El manejo de esta patología se ha descrito mediante técnicas farmacológicas y quirúrgicas, siendo más estudiadas las técnicas quirúrgicas, dentro de las que se encuentran la histeroscopía, laparoscopia, laparoscopia asistida con histeroscopía, laparotomía y el abordaje vaginal. Se realizó una revisión en PubMed-MEDLINE y LILACS respecto al manejo de este defecto, tomándose artículos publicados en los últimos cinco años, en la cual se encontraron 37 artículos. El manejo depende principalmente de factores como la sintomatología de la paciente, si tiene paridad satisfecha o no, la profundidad del defecto y las preferencias de la paciente. MÉD.UIS.2019;32(3):11-7

Palabras claves: Cesárea. Cicatriz. Histeroscopía. Laparoscopia.

\section{Therapeutic options for cesarean scar defect}

\section{Abstract}

The postcesarean scar defect is a defect in the anterior wall of the myometrium secondary to the cesarean section. Its prevalence is variable, reaching up to $84 \%$ in some studies, increasing with the number of previous cesarean sections. Most of the patients can be asymptomatic, but when it manifests, is mostly with abnormal uterine bleeding. Its management has been described with pharmacological and surgical techniques, the latter being the most researched. They include the hysteroscopy, laparoscopy, laparotomy, and the vaginal approach. A revision in PubMed-MEDLINE and LILACS about the management of this defect was done, including articles published in the last five years, resulting in 37 articles. The selection of the treatment depends on different factors such as the patient's symptoms, if she has satisfied parity or not, the depth of the defect and the patient's preferences. MÉD.UIS.2019;32(3):11-7

Keywords: Cesarean section. Cicatrix. Hysteroscopy. Laparoscopy.

¿Cómo citar este artículo?: Carreño-Martínez AC, Sepúlveda-Agudelo J. Opciones terapéuticas para el defecto de cicatriz uterina. MÉD. UIS.2019;32(3): 11-7. doi: 10.18273/revmed.v32n3-2019001 


\section{Introducción}

La cesárea es una de las intervenciones quirúrgicas más utilizadas en el mundo, con tasas que han ido en ascenso con el paso de los años. En Colombia, las tasas aumentaron del $24,9 \%$ en 1998 a 46,4\% en el $2015^{1,2}$. En otros países el panorama es similar, por ejemplo, en Estados Unidos la tasa aumentó del 20,7\% en 1996 al 32\% en 2015, y en Brasil aumentó del 15\% en 1970 a casi el $80 \%$ en el sector privado en $2004^{3-5}$.

Todas estas cifras se encuentran por encima de lo sugerido por la OMS, que es entre el 10 y el $15 \%$. Si bien la cesárea es un procedimiento muy benéfico y en algunos casos incluso necesario, puede generar en las pacientes secuelas permanentes como el defecto de cicatriz uterina, istmocele o nicho, el cual se define como un saco o una indentación en la cara anterior del miometrio, específicamente en el sitio de la cicatriz, que usualmente es en el istmo, sin datos específicos en cuanto al tamaño exacto que debe tener la lesión para ser diagnosticada ${ }^{7}$. Algunos autores refieren una clasificación del defecto según su profundidad, clasificándolo como grande cuando el miometrio residual es menor de $2,2 \mathrm{~mm}$ o 2,5mm, dependiendo si se diagnostica con ecografía transvaginal o histerosonografía, respectivamente ${ }^{3}$.

La prevalencia de estos defectos varía según diferentes factores, como la población, los criterios diagnósticos y la prueba diagnóstica utilizada; por ejemplo, el estudio de Van der Voet y et al., encontró una prevalencia del $75 \%$ en mujeres asintomáticas con antecedente de cesárea ${ }^{8}$. Según la prueba diagnóstica se han descrito prevalencias entre el 24 al $70 \%$ con ecografía transvaginal y del 56 al $84 \%$ con histerosonografía ${ }^{3}$. También se ha reportado el aumento de la prevalencia según el número de procedimientos realizados, llegando hasta el $100 \%$ en pacientes con tres cesáreas previas 3 . En población colombiana, se encontró un estudio realizado en el Hospital de San José de Bogotá, donde se documentó con histeroscopía una prevalencia del $83,3 \%$ en 42 pacientes con antecedentes de cesárea ${ }^{9}$.

Se han identificado algunos factores de riesgo, unos modificables y otros que dependen de la duración del trabajo de parto y de la técnica quirúrgica (Ver Tabla 1). El cuadro clínico es muy variado, en la mayoría de los casos son asintomáticas y el hallazgo del defecto es incidental, sin embargo, cuando hay manifestaciones clínicas, estas pueden ser sangrado uterino anormal, generalmente posmenstrual; dismenorrea, dispareunia, dolor pélvico crónico, infertilidad o incluso se puede manifestar con un embarazo ectópico en la cicatriz. El sangrado uterino anormal se presenta entre el 28,9 al $85,7 \%$ de los $\operatorname{casos}^{3,7,9,10}$. En el estudio realizado en el Hospital San José de Bogotá se describió una prevalencia de hemorragia uterina anormal en el $85,7 \%$, dismenorrea en el $61,9 \%$, dispareunia en el $40,5 \%$, dolor pélvico crónico en el $33,3 \%$ e infertilidad en un 7,1\% .

\begin{tabular}{l} 
Tabla 1. Factores de riesgo para el defecto de cicatriz uterina \\
\hline No modificables \\
- Edad materna $\leq 30$ años * \\
- Útero en retroverso * \\
\hline Relacionados con el trabajo de parto \\
- Duración $>5$ horas * \\
- Cesárea con dilatación cervical $\geq 5 \mathrm{~cm}$ * \\
- Baja estación al momento de la cesárea * \\
- Uso de oxitocina * \\
\hline Relacionados con la técnica quirúrgica \\
- Incisión cervical * \\
- Exclusión del endometrio * \\
- Cierre en un solo plano ** \\
- Suturas de absorción retardada ** \\
- Cierre muy isquémico ** \\
\hline * Evidencia respaldada \\
** Sugerido, pero con evidencia insuficiente \\
\hline
\end{tabular} Fuente: Tower AM, $2013^{10}$.

Como se mencionó anteriormente, el diagnóstico se puede realizar mediante diferentes métodos imagenológicos, generalmente el más usado es la ecografíatransvaginal, sinembargo, también sepuede observar el defecto mediante histerosonografía, histerosalpingografía, histeroscopía o resonancia nuclear magnética ${ }^{10}$.

Para el tratamiento se han descrito múltiples manejos, desde los farmacológicos hasta quirúrgicos, sin embargo, no se ha establecido del todo cuál de estas técnicas es la más apropiada. Considerando entonces que es una patología bastante frecuente y que puede llegar a tener una carga de enfermedad importante para las pacientes, se hizo necesario realizar una revisión de diferentes manejos planteados y sus diferencias en cuanto a mejoría en la sintomatología, complicaciones, ventajas y desventajas, y establecer aquellas condiciones que orientarían hacia la selección del manejo más adecuado. 


\section{Metodología de búsqueda}

Se realizó una búsqueda bibliográfica en las bases de datos PubMed-MEDLINE y LILACS con los términos MeSH Cesarean section, Cicatrix, Laparoscopy e Hysteroscopy, Laparotomy, y Vagina, y los términos DeCS Cesárea, Cicatriz, Laparoscopia, Histeroscopía, Laparotomía y Vagina en diferentes combinaciones. En la búsqueda se obtuvieron 604 artículos, posteriormente se seleccionaron aquellos publicados en los últimos cinco años, en idioma español o inglés. Finalmente, se hizo una revisión de títulos y resumen y se excluyeron aquellos que estuvieran repetidos y todos aquellos cuyo tema fuera diferente al manejo de los defectos de cicatriz uterina, obteniendo un número final de 37 artículos. Se adicionaron 7 artículos más para contextualización del tema en la introducción.

\section{Resultados}

\section{Manejo farmacológico}

\section{Anticonceptivos orales bihormonales}

Se ha descrito el uso de esquemas tanto continuos como cíclicos. En los esquemas continuos se usan los anticonceptivos orales durante 21 días y se descansan 7; mientras que en los cíclicos, los anticonceptivos orales se usan solo durante los primeros 5 días de cada ciclo ${ }^{11,12}$. En cuanto a la mejoría de los síntomas, un estudio reportó una disminución del dolor pélvico en el $33,3 \%$ y del sangrado en el $4,3 \%$, sin embargo, otros estudios han reportado una mejoría de los síntomas hasta en el $91 \%$ de los $\operatorname{casos}^{11-13}$. Hasta el momento no se ha estudiado la diferencia en la eficacia entre estos dos esquemas.

Diferentes estudios han mostrado una disminución del tamaño del defecto y de su vascularización $n^{7,13,14}$; sin embargo, el estudio realizado por Wu et al., no encontró un cambio en el defecto posterior al tratamiento durante un año, por lo que recomendaron la resección quirúrgica sobre el manejo hormonal debido a una mejor calidad de vida en las pacientes y menos necesidad de consultas ${ }^{11}$. Por otro lado existe el riesgo de retornar a la condición clínica previa una vez se suspenda el tratamiento ${ }^{11,12,15}$.

La indicación principal para la selección del manejo con estos medicamentos es en pacientes sintomáticas que no estén planeando un embarazo a corto plazo o que no deseen la realización de una cirugía ${ }^{11,12}$.
Opciones terapéuticas para el defecto de cicatriz uterina Dispositivo intrauterino liberador de levonorgestrel

En el estudio de Zhang et al., se evaluó el efecto de este dispositivo, sin embargo, durante el seguimiento de seis meses no se evidenciaron cambios en la duración de la menstruación. Los autores atribuyeron esto al corto tiempo de seguimiento y a posibles efectos secundarios del dispositivo en los meses iniciales ${ }^{16}$. No se encontraron otros estudios que evaluaran los efectos de este manejo.

\section{Manejo quirúrgico}

\section{Histeroscopía}

En el estudio de Li et al., se describió un doble propósito con la corrección mediante histeroscopía: mejorar el drenaje menstrual evitando el acúmulo de sangre, y lograr la coagulación de vasos dilatados en la superficie del defecto o de glándulas endometriales para disminuir la producción de sangre $^{17}$. Este procedimiento ha mostrado mejoría o resolución de los síntomas, principalmente del sangrado posmenstrual y el dolor pélvico, en una gran proporción de pacientes entre el 59 al 100\% y $87 \%$ al $100 \%$, respectivamente ${ }^{3,18-21}$. Adicionalmente, en el estudio de Cohen et al., se evaluó la efectividad y seguridad de una segunda histeroscopía en ocho pacientes con histeroscopía previa fallida; considerada como persistencia de sangrado posmenstrual durante tres meses después del procedimiento o como reaparición del istmocele en el primer año de seguimiento. En este estudio se encontró que la reintervención logró una mayor reducción de los días de sangrado comparado con la intervención previa y no se presentaron complicaciones ${ }^{22}$.

Gubbini et al., estudiaron a 41 pacientes con infertilidad y diagnóstico de istmocele, las cuales presentaban sangrado posmenstrual, y 19 presentaban dolor pélvico en los últimos tres a ocho años; después de hacer la corrección del defecto mediante histeroscopía se encontró que todas lograron una gestación en los 12 a 24 meses siguientes a la cirugía, de estas, el 9,8\% tuvo un aborto espontáneo en el primer trimestre y el 90,2\% llevó el embarazo a término, finalizando con un parto por cesárea ${ }^{19}$.También se ha reportado en otros estudios una tasa del 50 al 96,1\% de embarazos posterior a la corrección mediante esta técnica quirúrgica3,23,24. Cohen et al., también evaluaron los resultados 
obstétricos en pacientes con dos procedimientos histeroscópicos para resección del defecto, encontrando que después de la primera cirugía el $50 \%$ de las pacientes lograron un embarazo, y el $33 \%$ lograron embarazo con la segunda histeroscopía, además presentaron un parto a término mediante cesárea a las 38 semanas $^{22}$.

Las complicaciones durante 0 después del procedimiento son muy poco frecuentes, sin embargo, no hay datos claros. Algunas de las posibles complicaciones descritas en la literatura son: hemorragias, infecciones, adherencias endometriales, ruptura uterina, fístula vesicouterina, alteraciones de la implantación placentaria y dehiscencia de la cicatriz, teniendo un poco más de riesgo para la lesión de órgano vecino si la pared miometrial es muy delgada $4,7,12,17,18,20$. Algunas de las ventajas que se destacan son: un menor tiempo operatorio, menor estancia hospitalaria, menor pérdida sanguínea y menor gasto económico ${ }^{16}$.

En el estudio de Vervoort et al., se evaluó el grosor miometrial posterior a la intervención y no se encontró un aumento de este en comparación con el grupo sin dicha intervención, por lo que no se debería realizar la intervención si el miometrio es muy delgado ya que aumentaría el riesgo de ruptura uterina en una siguiente gestación ${ }^{4}$. Sin embargo, en el estudio de Tsuji et al., se encontró un aumento del grosor miometrial posterior a la histeroscopía de 2,1mm a 4,2mm en 16 de las 18 pacientes con defecto de cicatriz uterina ${ }^{25}$.

En general, este procedimiento se recomienda en pacientes sintomáticas con útero en anteversoflexión, en pacientes con un miometrio residual $\geq 3 \circ 3,5 \mathrm{~mm}$, cuyo defecto sea $<50 \%$ del grosor de la pared miometrial, o aquellas que tengan paridad satisfecha independiente de la profundidad del defecto $4,17,18,21,24,26$.

\section{Laparoscopia}

Se ha descrito mejoría de la sintomatología en el 60 al $100 \%$ de las pacientes a quienes se les realiza laparoscopia $3,12,17,24,27,28$. En un estudio realizado por Dosedla et al., en 11 pacientes con defecto de cicatriz uterina, el 63,6\% resultó con total resolución de todos sus síntomas, mientras que el $36,4 \%$ persistió con dispareunia, $27,3 \%$ con dolor pélvico crónico y $18,2 \%$ con sangrado posmenstrual y dismenorrea ${ }^{29}$. En el estudio de Donnez et al., 34 pacientes presentaron mejoría, una de las pacientes que tenía tres cesáreas previas presentó empeoramiento del dolor y de la dismenorrea posterior a la cirugía, evidenciándose sangre en sitio del defecto y un aumento del tamaño del mismo por lo cual requirió histerectomía laparoscópica; tres pacientes requirieron una segunda intervención, la cual se realizó por histeroscopía ${ }^{30}$.

Se ha reportado una prevalencia de embarazos posterior a la corrección del 23,5 al 55,6\% 3,16,17,24. En un estudio realizado por Zhang et al., se observó una prevalencia de $37,5 \%$, las pacientes presentaron un parto pretérmino, siete partos a término y un embarazo ectópico en la cicatriz uterina. Tres de las pacientes estaban embarazadas en el momento de culminado el estudio ${ }^{16}$. Por otra parte, el estudio de Marotta et al., encontró una prevalencia del 30,7\% de embarazo espontáneo posterior a la corrección, los cuales se desarrollaron sin complicaciones ${ }^{(28)}$. Por último, en el estudio de Tanimura et al., se evidenció una prevalencia del $30 \%$ de partos pretérmino en pacientes con partos a término previos, lo cual asociaron a un acortamiento cervical por remoción del tejido fibroso de la cicatriz ${ }^{24}$.

No se han descrito hasta el momento complicaciones relacionadas con el procedimiento ${ }^{13,29,31,32}$. Algunas de las desventajas son los mayores costos, mayor tiempo operatorio, mayor pérdida sanguínea y mayor tiempo de estancia hospitalaria. Por otra parte, las ventajas descritas son la posibilidad de utilizarlo en defectos muy profundos y la posibilidad de tratar defectos que tengan una localización alta, mejor visualización del defecto, menor riesgo de daño a órgano vecino y una corta estancia hospitalaria ${ }^{16,33}$.

Como ya se mencionó anteriormente, esta técnica permite una mejor visualización del defecto, lo que facilita un abordaje más completo del mismo. Es por esto, que se ha observado una restauración del miometrio residual, reportándose un aumento del grosor de hasta $9 \mathrm{~mm}^{7,12,14,16,17,27}$. Considerando esos hallazgos, su indicación en la literatura es en pacientes sintomáticas con miometrio residual $<3$ $-3,5 \mathrm{~mm}$, o con un defecto que comprometa $\geq 50 \%$ de la pared miometrial con síntomas severos y sin paridad satisfecha $4,17,26,31,34$. En el estudio de Tanimura et al., se añadió una indicación en caso de que la paciente tenga un útero en retroversoflexión, debido a su mayor riesgo de defectos de la cicatriz ${ }^{24}$. Adicionalmente, se han descrito correcciones asistidas por robótica con resultados favorables, sin 
Septiembre - Diciembre

embargo, no hay suficientes datos, posiblemente por el alto costo que conlleva este procedimiento ${ }^{14,35}$.

\section{Abordaje vaginal}

Se ha descrito que entre el $85,9 \%$ y el $93,5 \%$ de las pacientes presenta mejoría de su sintomatología 3,12,14,36,37. En un estudio realizado por Zhou et al., se reportaron como factores asociados a un buen pronóstico sangrado menstrual $<10$ días un grosor del miometrio residual $\geq 8,5 \mathrm{~mm}$ seis meses después de la cirugía y que el tiempo entre la cesárea y la corrección mediante abordaje vaginal fuera menor o igual a dos años ${ }^{38}$. Se han reportado tasas del $22 \%$ de embarazos posterior a la corrección ${ }^{13}$.

Con respecto a las ventajas, se ha visto un tiempo operatorio mucho menor, menor pérdida sanguínea, menor estancia hospitalaria y menores costos, comparado con las otras técnicas, pero tiene una limitación cuando los defectos tienen una localización más alta ${ }^{3,16,36,39}$. En cuanto a las complicaciones, en el estudio de Zhou et al., se reportaron pequeños hematomas en el 2,5\% de las pacientes, mientras que en el estudio de Luo et al.,se reportó un 2,4\% de infección posoperatoria por bacilos gramnegativos, la cual resolvió con manejo antibiótico. Otra posible complicación sería, al igual que en las demás técnicas, la lesión de órgano vecino ${ }^{38,39}$.

Al igual que con la laparoscopia, el abordaje vaginal ha logrado una restauración del grosor del miometrio residual alcanzando un aumento de hasta $6 \mathrm{~mm}$ y una disminución de la profundidad de la cicatriz de hasta $7 \mathrm{~mm}$, según el estudio de Zhou et al.,38. Otro estudio encontró una reducción significativa o resolución completa del defecto en el $87 \%$ de las pacientes, sin diferencia significativa con la laparoscopia ${ }^{37}$.

No se encuentran indicaciones claras para la selección de esta intervención, sin embargo, el estudio de Zhang comparó la técnica laparoscópica con el abordaje vaginal y concluyó que, en cuanto a efectividad terapéutica, son comparables, destacando los menores costos y menor tiempo operatorio del abordaje vaginal ${ }^{37}$.

\section{Minilaparotomía}

En el estudio de Pomorski et al., se consideraron candidatas a corrección mediante minilaparotomía pacientes sintomáticas, con rechazo a la terapia hormonal, y con un grosor miometrial residual $\leq 2,2 \mathrm{~mm}$. Este último criterio fue seleccionado en
Opciones terapéuticas para el defecto de cicatriz uterina

base a que defectos menos profundos podrían beneficiarse de una cirugía menos invasiva, como la histeroscopía ${ }^{40}$.

Se ha observado una mejoría de los síntomas en el 60 al $100 \%$ de las pacientes ${ }^{40,41}$. En el estudio de Schepker et al., se observó una tasa de embarazos del $60 \%$, dos de los embarazos no tuvieron complicaciones, uno de los partos incluso fue vía vaginal. El otro embarazo, tuvo un abrupcio de placenta que requirió cesárea de emergencia ${ }^{41}$. Otros estudios han encontrado una tasa del $71 \%$ de embarazos posterior a la corrección ${ }^{13}$. En cuanto a las complicaciones, se ha reportado en dos pacientes hematuria transitoria en el posoperatorio, que mejoró sin tratamiento ${ }^{40}$. No se han descrito otras complicaciones intra o posoperatorias ${ }^{13,40,41}$.

En el estudio de Pomorski et al., el grosor miometrial antes y tres meses después de la cirugía fue de $1,9 \mathrm{~mm}$ y $8 \mathrm{~mm}$ respectivamente, sin embargo, en el $28,5 \%$ hubo una persistencia del defecto, con una profundidad de $4,4-4,5 \mathrm{~mm}^{40}$.

\section{Laparoscopia combinada con histeroscopía}

En un estudio de Li et al., se utilizó esta técnica en 40 pacientes, la cual reportó una mejoría de los síntomas en el $90 \%$ de las pacientes seis meses después de la cirugía, sin embargo, ellos encontraron que en el 7,5\% de las pacientes persistió el defecto, aún después de la corrección ${ }^{15}$. En el estudio de Liu et al., se reportó una mejoría en los síntomas del 89,8\%, y según la evaluación anatómica a los seis meses mediante ecografía, el 95,9\% de los procedimientos fue efectivo ${ }^{42}$.

Otro estudio realizado por $\mathrm{Wu}$ et al., evidenció un retorno a la normalidad de la menstruación en el $24,5 \%$, aumento en el $2 \%$ y el $73,5 \%$ restante no presentó cambios. Además, se reportó resolución del dolor abdominal en el 15,8\%, una mejoría significativa en el $68,4 \%$ y el $31,6 \%$ persistió sin cambios. En cuanto a la condición de la cicatriz posterior a la cirugía, se evidenció una resolución total del defecto en el $73,5 \%$, disminución significativa del $20,4 \%$ y en el $6,1 \%$ no se presentó ningún cambio ${ }^{11}$.

En cuanto a la tasa de embarazos posterior a la cirugía no se encuentran muchos datos, solo en dos estudios se encontró una tasa del $4 \% \%^{11,42}$. En un reporte de Masuda et al., se describió una paciente que requirió un abordaje combinado, ya que, aún cuando se había 
visualizado en una resonancia nuclear magnética, con la laparoscopia no se pudo evidenciar el defecto por lo que se requirió de la histeroscopía guiada por laparoscopia para resecar el mismo. En este caso, la paciente tenía una historia de infertilidad de tres años y sangrado posmenstrual, y después de la intervención el sangrado resolvió por completo y la paciente pudo concebir en dos ocasiones con tres intentos de inseminación intrauterina ${ }^{43}$.

Las ventajas descritas son la mejor visualización de la cavidad abdominopélvica por la laparoscopia, la mejor ubicación del defecto a través de una luz en el histeroscopio, la posibilidad de evaluar la reparación mediante histeroscopía inmediatamente posterior a la corrección y la posibilidad de separar la vejiga de la pared uterina anterior para evitar el riesgo de iatrogenia $15,37,44$. Por otra parte, las desventajas descritas son el mayor tiempo quirúrgico, mayores costos y el requerimiento de un cirujano que esté familiarizado con ambas técnicas ${ }^{15,42}$. Algunas de las complicaciones descritas que pueden estar asociadas a este tipo de intervención son la hemorragia masiva, lesión de órgano vecino y la perforación uterina, sin embargo, en los estudios evaluados, no se presentaron ${ }^{11,15,42}$.

\section{Conclusiones}

El defecto de cicatriz uterina es una condición que se debe tener en cuenta en pacientes con antecedentes de cesárea y que presenten sangrado posmenstrual, dismenorrea, dolor pélvico crónico, dispareunia o infertilidad. El manejo solo se debe recomendar en pacientes sintomáticas, así tengan o no paridad satisfecha. El uso de anticonceptivos orales se podría recomendar en pacientes que no deseen una intervención quirúrgica y no estén planeando un embarazo. En cuanto al manejo quirúrgico, si el defecto no compromete notoriamente el grosor del miometrio (grosor residual $\geq 3-3,5 \mathrm{~mm}$ ) o si la paciente tiene paridad satisfecha se podría recomendar la histeroscopía; pero si el grosor miometrial es muy delgado (grosor residual $<3-3,5 \mathrm{~mm}$ ) y la paciente desea concebir en el futuro se debería realizar una resección total del defecto con laparoscopia, laparotomía, un abordaje vaginal o usar la combinación de laparoscopia e histeroscopía.

Otra parte importante a considerar es la prevención, por lo que se debería restringir el uso de la cesárea y reservar su uso únicamente en casos donde esté realmente indicada, ya que, aunque en la mayoría de casos es una entidad asintomática, el defecto de cicatriz uterina puede tener un amplio rango de manifestaciones que pueden llegar a comprometer seriamente el bienestar de la paciente.

\section{Referencias bibliográficas}

1. Rubio-Romero JA, Fonseca-Pérez JE, Molina S, Buitrago Leal M, Zuleta JJ, Ángel-Müller E, et al. Racionalización del uso de la cesárea en colombia. Consenso de la federación colombiana de obstetricia y ginecología (fecolsog) y la federación colombiana de perinatología (fecopen). Bogotá, 2014. Rev Colomb Obstet Ginecol. 2014;65(2):139-51.

2. Departamento Administrativo Nacional de Estadística (DANE). Nacimientos 2015. Cuadro 4. Nacimientos por tipo de parto según departamento de ocurrencia y sitio del parto. 2017.

3. Tulandi T, Cohen A. Emerging manifestations of cesarean scar defect in reproductive-aged women. J Minim Invasive Gynecol. 2016;23(6):893-902.

4. Vervoort A, Van der Voet L, Hehenkamp W, Thurkow A, van Kesteren P, Quartero H, et al. Hysteroscopic resection of a uterine caesarean scar defect (niche) in women with postmenstrual spotting: a randomised controlled trial. BJOG An Int J Obstet Gynaecol. 2018;125(3):326-334.

5. Martin JA, Hamilton BE, Osterman MJKS, Driscoll AK, Mathews TJ. National Vital Statistics Reports. 2017;66(1).

6. Organización Panamericana de la Salud, Organización Mundial de la Salud. La cesárea solo debería realizarse cuando sea medicamente necesaria. 2015.

7. Sipahi S, Sasaki K, Miller CE. The minimally invasive approach to the symptomatic isthmocele - what does the literature say? A step-by-step primer on laparoscopic isthmocele - excision and repair. Curr Opin Obstet Gynecol. 2017;29(4):257-65.

8. van der Voet L (Lucet) F, Limperg T, Veersema S, Timmermans A, Bij de Vaate AMJ, Brölmann HAM, et al. Niches after cesarean section in a population seeking hysteroscopic sterilization. Eur J Obstet Gynecol Reprod Biol. 2017;214:104-8.

9. Benedetti O, Agudelo C, Rodríguez R, Miranda Á, Rodríguez H, Castro C. Prevalencia de istmocele en el Hospital de San José de Bogotá, Colombia. Rev Chil Obstet Ginecol. Sociedad Chilena de Obstetricia y Ginecología; 2016 Dec;81(6):465-72.

10. Tower AM, Frishman GN. Cesarean Scar Defects: An Underrecognized Cause of Abnormal Uterine Bleeding and Other Gynecologic Complications. J Minim Invasive Gynecol. 2013;20(5):562-72.

11. Wu X, Lin R, Yuan Y, He Y, Xu C, Hu W, et al. A comparison of the effects of two different treatment methods on cesarean section scar diverticulum: The surgical repair of cesarean scars via combined laparoscopy and hysteroscopy vs. cyclic estrogen/ progesterone therapy. Int J Clin Exp Med. 2017;10(4):7144-50

12. Van Der Voet LF, Vervoort AJ, Veersema S, Bijdevaate AJ, Brölmann HAM, Huirne JAF. Minimally invasive therapy for gynaecological symptoms related to a niche in the caesarean scar: A systematic review. BJOG An Int J Obstet Gynaecol. 2014;121(2):145-56.

13. Setubal A, Alves J, Osório F, Guerra A, Fernandes R, Albornoz J, et al. Treatment for Uterine Isthmocele, a Pouch-Like Defect at the Site of Cesarean Section Scar. J Minim Invasive Gynecol. 2018;25(1):38-46.

14. Futyma K, Gałczyński K, Romanek K, Filipczak A, Rechberger $\mathrm{T}$. When and how should we treat cesarean scar defect isthmocoele? Ginekol Pol. 2016;87(9):664-8.

15. Li C, Tang S, Gao X, Lin W, Han D, Zhai J, et al. Efficacy of Combined Laparoscopic and Hysteroscopic Repair of PostCesarean Section Uterine Diverticulum: A Retrospective Analysis. Biomed Res Int. 2016;2016(5):1-6.

16. Zhang X, Yang M, Wang Q, Chen J, Ding J, Hua K. Prospective evaluation of five methods used to treat cesarean scar defects. Int J Gynaecol Obstet. 2016;134(3):336-9.

17. Li C, Guo Y, Liu Y, Cheng J, Zhang W. Hysteroscopic and laparoscopic management of uterine defects on previous cesarean delivery scars. J Perinat Med. 2014;42(3):363-70.

18. Feng Y-L, Li M-X, Liang X, Li X-M. Hysteroscopic Treatment 


\section{Septiembre - Diciembre}

of Postcesarean Scar Defect. J Minim Invasive Gynecol. 2012;19(4):498-502.

19. Gubbini G, Centini G, Nascetti D, Marra E, Moncini I, Bruni L, et al. Surgical Hysteroscopic Treatment of Cesarean-Induced Isthmocele in Restoring Fertility: Prospective Study. J Minim Invasive Gynecol. 2011;18(2):234-7.

20. Raimondo G, Grifone G, Raimondo D, Seracchioli R, Scambia G, Masciullo V. Hysteroscopic Treatment of Symptomatic Cesareaninduced Isthmocele: A Prospective Study. J Minim Invasive Gynecol. 2015;22(2):297-301.

21. Muzii L, Domenici L, Lecce F, Feliciantonio MDI, Frantellizzi R, Marchetti C, et al. Clinical outcomes after resectoscopic treatment of cesarean-induced isthmocele: a prospective case-control study. Eur Rev Med Pharmacol Sci. 2017;21(15):3341-3346.

22. Cohen SB, Mashiach R, Baron A, Goldenberg M, Schiff E, Orvieto R, et al. Feasibility and efficacy of repeated hysteroscopic cesarean niche resection. Eur J Obstet Gynecol Reprod Biol. 2017;217:12-7.

23. Florio P, Filippeschi M, Moncini I, Marra E, Franchini M, Gubbini G. Hysteroscopic treatment of the cesarean-induced isthmocele in restoring infertility. Curr Opin Obstet Gynecol. 2012;24(3):180-6.

24. Tanimura S, Funamoto H, Hosono T, Shitano Y, Nakashima M, Ametani Y, et al. New diagnostic criteria and operative strategy for cesarean scar syndrome: Endoscopic repair for secondary infertility caused by cesarean scar defect. J Obstet Gynaecol Res. 2015;41(9):1363-9.

25. Tsuji S, Kimura F, Yamanaka A, Hanada T, Hirata K, Takebayashi A, et al. Impact of hysteroscopic surgery for isthmocele associated with cesarean scar syndrome. J Obstet Gynaecol Res. 2018 Jan;44(1):43-48.

26. Brown K, Tkacz Z. Hysteroscopic and laparoscopic management of caesarean scar (niche) defects in symptomatic patients. J Obstet Gynaecol Res. 2018; 38(5):730.

27. Api M, Boza A, Gorgen H, Api O. Should cesarean scar defect be treated laparoscopically? A case report and review of the literature. J Minim Invasive Gynecol. 2015;22(7):1145-52.

28. Marotta ML, Donnez J, Squifflet J, Jadoul P, Darii N, Donnez O. Laparoscopic repair of post-cesarean section uterine scar defects diagnosed in nonpregnant women. J Minim Invasive Gynecol. 2013;20(3):386-91.

29. Dosedla E, Calda P. Outcomes of laparoscopic treatment in women with cesarean scar syndrome. Med Sci Monit. 2017:23:4061-6.

30. Donnez O, Donnez J, Orellana R, Dolmans MM. Gynecological and obstetrical outcomes after laparoscopic repair of a cesarean scar defect in a series of 38 women. Fertil Steril. 2017;107(1):28996.

31. Giorgio A, Buggio L, Berlanda N, Vercellini P. Laparoscopic repair of a symptomatic post cesarean isthmocele: a video case report. Fertil Steril. 2017;107(6):e17-e18.

32. Urman B, Arslan T, Aksu S, Taskiran C. Laparoscopic Repair of
Cesarean Scar Defect "Isthmocele." J Minim Invasive Gynecol. 2016;23(6):857-858.

33. Ciebiera M, Jakiel G, Sałbuszewska-Jóźwiak A. Laparoscopic correction of the uterine muscle loss in the scar after a Caesarean section delivery. Wideochir Inne Tech Maloinwazyjne. 2013;8(4):342-345

34. Bakavičiūtė G, Špiliauskaitẻ S, Meškauskienẻ A, Ramašauskaitė D. Laparoscopic repair of the uterine scar defect - successful treatment of secondary infertility: a case report and literature review. Acta Med Litu. 2016;23(4):227-231.

35. Mahmoud MS, Nezhat FR. Robotic-assisted laparoscopic repair of a cesarean section scar defect. J Minim Invasive Gynecol. 2015;22(7):1135-6.

36. Chen Y, Chang Y, Yao S. Transvaginal management of cesarean scar section diverticulum: a novel surgical treatment. Med Sci Monit. 2014:20:1395-9.

37. Zhang Y. A Comparative Study of Transvaginal Repair and Laparoscopic Repair in the Management of Patients With Previous Cesarean Scar Defect. J Minim Invasive Gynecol. 2016;23(4):53541.

38. Zhou J, Yao M, Wang H, Tan W, Chen P, Wang X. Vaginal Repair of Cesarean Section Scar Diverticula that Resulted in Improved Postoperative Menstruation. J Minim Invasive Gynecol. 2016;23(6):969-78.

39. Luo L, Niu G, Wang Q, Xie HZ, Yao SZ. Vaginal Repair of Cesarean Section Scar Diverticula. J Minim Invasive Gynecol. 2012;19(4):454-8.

40. Pomorski M, Fuchs T, Rosner-Tenerowicz A, Zimmer M. Sonographic evaluation of surgical repair of uterine cesarean scar defects. J Clin Ultrasound [Internet]. 2017;45(8):455-60. Disponible en: https://www.ncbi.nlm.nih.gov/pubmed/28186617

41. Schepker N, Garcia-Rocha G-J, von Versen-Höynck F, Hillemanns P, Schippert C. Clinical diagnosis and therapy of uterine scar defects after caesarean section in non-pregnant women. Arch Gynecol Obstet. 2015 Jun 17;291(6):1417-23. Disponible en: https://www.ncbi.nlm.nih.gov/pubmed/25516174

42. Liu SJ, Lv W, Li W. Laparoscopic repair with hysteroscopy of cesarean scar diverticulum. J Obstet Gynaecol Res [Internet]. 2016;42(12):1719-23. Disponible en: https://obgyn.onlinelibrary. wiley.com/doi/abs/10.1111/jog.13146

43. Masuda H, Uchida H, Maruyama T, Sato K, Sato S, Tanaka M, et al. Successful treatment of atypical cesarean scar defect using endoscopic surgery. BMC pregnancy childb [Internet]. 2015;15:342. Disponible en: https://www.ncbi.nlm.nih.gov/pmc/ articles/PMC4687144/

44. Nirgianakis K, Oehler R, Mueller M. The Rendez-vous technique for treatment of caesarean scar defects: a novel combined endoscopic approach. Surg Endosc [Internet]. 2016;30(2):770-1. Disponible en: https://www.ncbi.nlm.nih.gov/pubmed/26104791 